\title{
Radio Observations of Supernova 1987A
}

\author{
L. Staveley-Smith ${ }^{1,2}$, T. M. Potter ${ }^{1}$, G. Zanardo ${ }^{1}$, \\ B. M. Gaensler ${ }^{2,3}$ and C.-Y. $\mathbf{N g}^{4}$ \\ ${ }^{1}$ International Centre for Radio Astronomy Research, University of Western Australia, \\ Crawley, WA 6009, Australia \\ email: Lister.Staveley-Smith@icrar.org \\ ${ }^{2}$ ARC Centre of Excellence for All-sky Astrophysics (CAASTRO) \\ ${ }^{3}$ Sydney Institute of Astronomy, School of Physics, The University of Sydney, NSW 2006, \\ Australia \\ ${ }^{4}$ Department of Physics, The University of Hong Kong, Pokfulam Road, Hong Kong
}

\begin{abstract}
Supernovae and their remnants are believed to be prodigious sources of Galactic cosmic rays and interstellar dust. Understanding the mechanisms behind their surprisingly high production rate is helped by the study of nearby young supernova remnants. There has been none better in modern times than SN1987A, for which radio observations have been made for over a quarter of a century. We review extensive observations made with the Australia Telescope Compact Array (ATCA) at centimetre wavelengths. Emission at frequencies from 1 to $100 \mathrm{GHz}$ is dominated by synchrotron radiation from an outer shock front which has been growing exponentially in strength from day 3000, and is currently sweeping around the circumstellar ring at about $4000 \mathrm{~km} \mathrm{~s}^{-1}$. Three dimensional models of the propagation of the shock into the circumstellar medium are able to reproduce the main observational features of the remnant, and their evolution. We find that up to $4 \%$ of the electrons encountered by the shock are accelerated to relativistic energies. High-frequency ALMA observations will break new ground in the understanding of dust and molecule production.
\end{abstract}

Keywords. Supernovae, SN1987A, radio astronomy

\section{Overview}

As the brightest supernova since Kepler's of 1604, SN1987A has been a Rosetta Stone in our understanding of the physics of Type II supernova explosions. It was the first supernova with a known progenitor, and the brightest since the invention of the telescope. It has therefore allowed a number of unprecedented studies including: detailed comparison with models of the very final stages of stellar evolution; the detection of the first neutrinos from an extrasolar source, providing evidence for the formation of a neutron star (Vissani et al. 2010); measurement of absolute distance using the light travel time method (Panagia et al. 1991); the probing and analysis of the circumstellar medium excited by the ultraviolet flash; measurement of the radioactive decay products of the explosion; study of the interaction of the expanding blast wave with the anisotropic circumstellar medium laid out by the stellar winds from various stages of the progenitor star; and formation of dust and molecules in the cooling ejecta. The fact that, to date, over 2000 refereed papers have been written about SN1987A, or have mentioned SN1987A in their abstracts, is testament to the significance of the event.

In this paper, we discuss radio observations in the two and a half decades following the explosion. These observations are particularly sensitive to detailed shock physics, magnetic field amplification and circumstellar structure, and have allowed us to clearly 


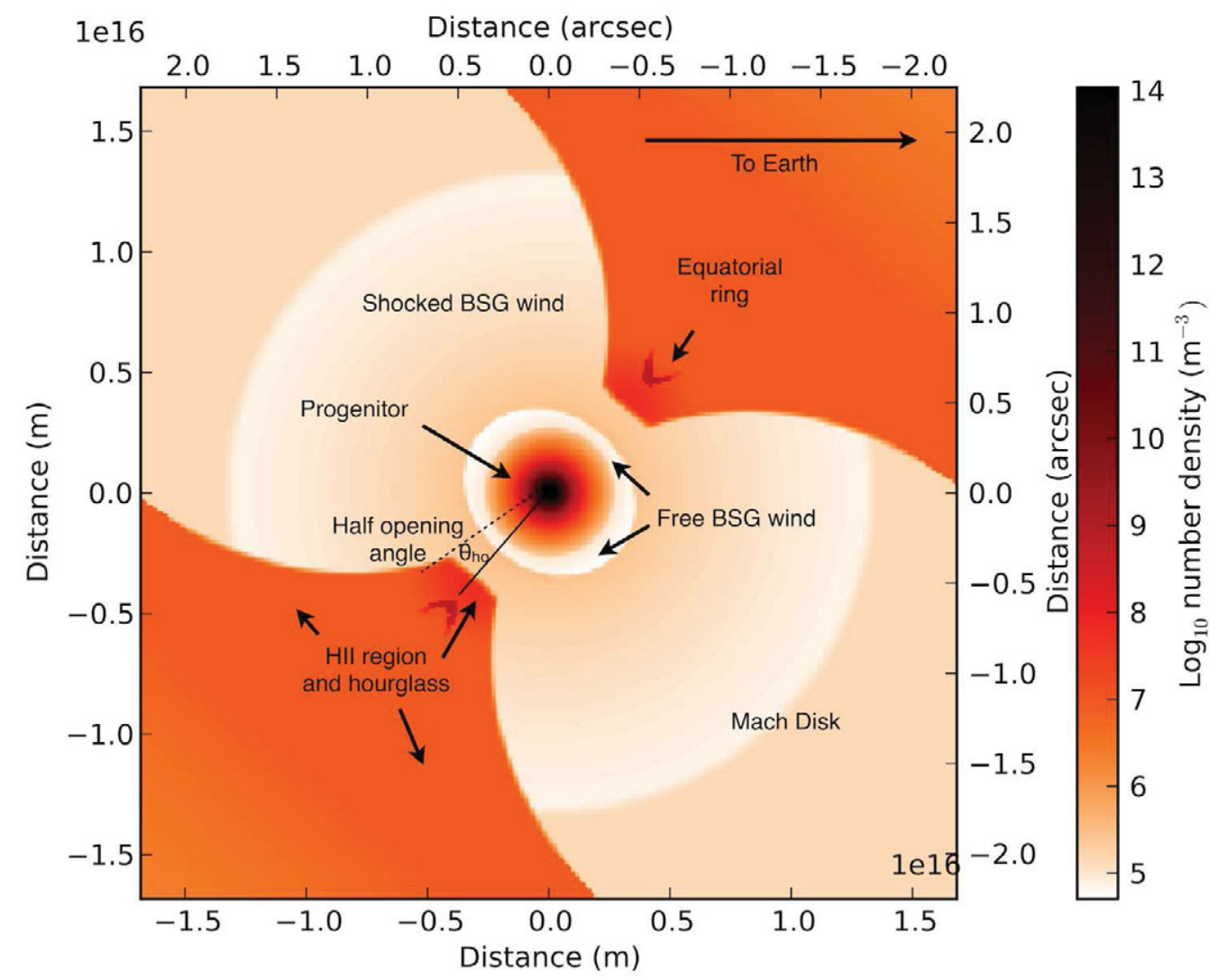

Figure 1. A model of the initial 'hourglass' environment prior to explosion. The environment was created by the progenitor star in its evolution from red supergiant to blue supergiant (BSG). The supernova shock is currently at the position of the equatorial ring the ring is perpendicular to the plane of the paper (Potter 2012). A Mach disk is formed at the point where the BSG wind again goes supersonic. The axes are labelled in arcsec and in units of $10^{16} \mathrm{~m}$.

follow the evolution of a Type II supernova into the important supernova remnant (SNR) phase.

\section{Environment}

The initial interaction of the SN1987A blast wave with the circumstellar medium resulted in the generation of a burst of synchrotron radiation lasting a few days (Turtle et al. 1987; Storey \& Manchester 1987). Radio (and X-ray) observations of other, more distant, type II supernovae have been used to compare progenitor mass-loss rates using the theoretical framework laid out by Chevalier (1982), and to probe the uniformity of the mass loss. Similar studies on SN1987A were consistent with the observation that the progenitor was a blue supergiant, with a fast but tenuous stellar wind at the time of explosion. However SN1987A was notable in that the luminosity of this initial radio burst, which peaked at about day 3, was around $10^{4}$ times weaker than powerful type II supernovae such as SN1993J. Nevertheless, following the discovery by the ESO New Technology Telescope (Wampler et al. 1990) and the HST of a ring-like structure around SN1987A, there was some expectation of a re-brightening of the radio emission. This was seen at day 1200 by the MOST and ATCA telescopes (Staveley-Smith et al. 1992). 


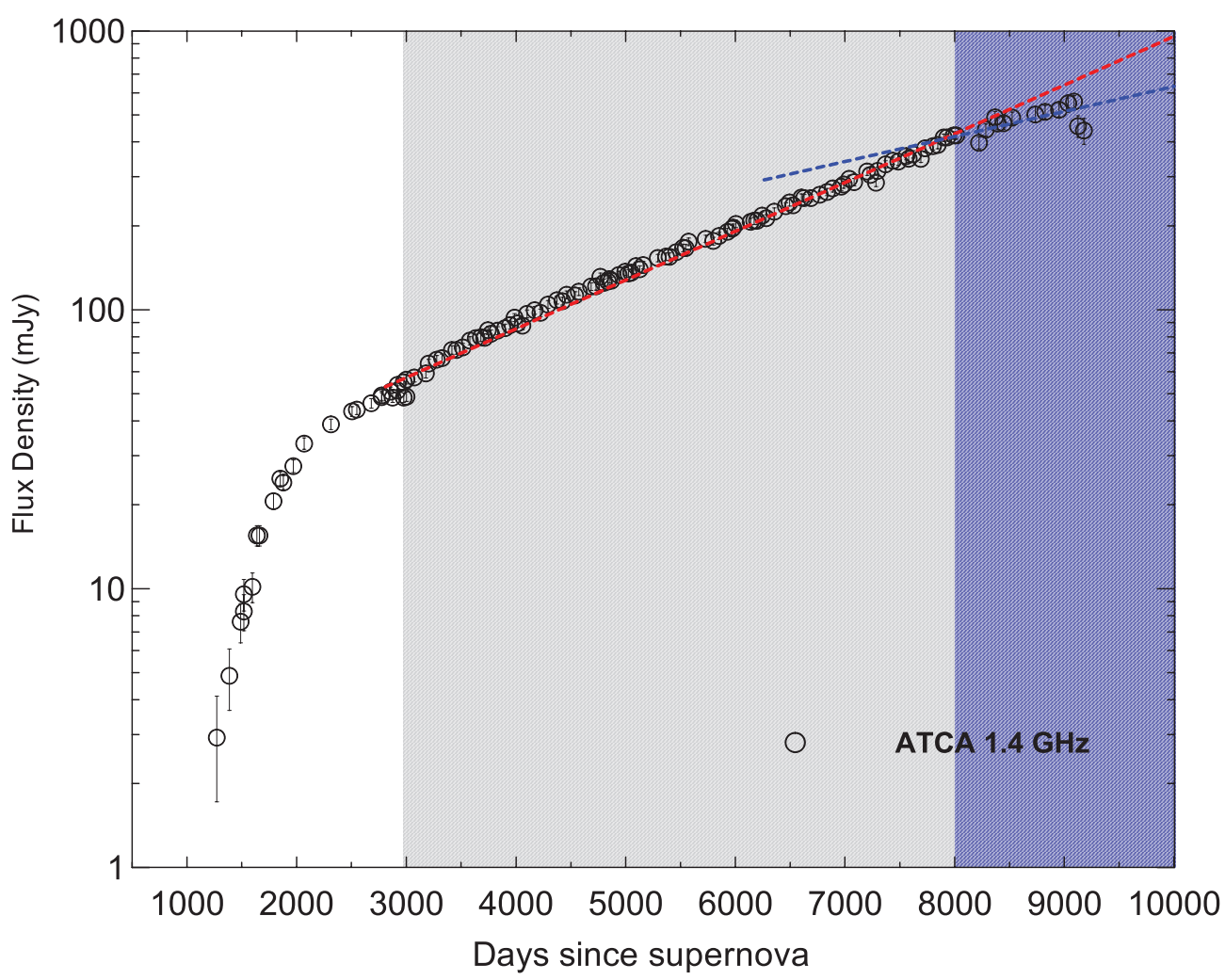

Figure 2. Radio observations at $1.4 \mathrm{GHz}$ have been conducted with ATCA at intervals of 4-6 weeks since the remnant was re-detected around day 1200. Its evolution is divided into three phases: (a) a linear phase up to day 3000; (b) an exponential phase to day 8000 (light shading); and (c) a slower exponential phase from day 8000 (darker blue shading). The red dotted line is the fit of Zanardo et al. (2010).

As a result of early optical and radio observations, and studies of the light echoes of the explosion itself (Crotts, Kunkel \& McCarthy 1989), a detailed picture of the environment around the supernova has been constructed. The main features of the circumstellar environment are illustrated in Fig. 1. The well-known equatorial ring, which defines the waist of an hourglass feature, is perpendicular to the plane of the paper. The northernmost edge of the ring is closest to Earth. Within the hourglass, there are (pre-supernova) regions which signify both freely expanding wind and shocked wind. It is at the interface of the two where it is believed that the first radio emission was generated (Chevalier \& Dwarkadas 1995).

\section{Evolution}

After re-emergence of the radio remnant at day 1200, the flux density was seen to increase linearly with time until day 4500, when small departures were evident (Manchester et al. 2002). In datasets from day 3000 to 8000 , a better description is an exponential increase (Zanardo et al. 2010), perhaps reflecting the exponential nature of the particle acceleration process and/or the increasing area of the expanding shock front. Observational data from the ATCA at $1.4 \mathrm{GHz}$ is shown in Fig. 2. Similar exponential evolution was seen in the X-rays, though the detailed temporal behaviour has been quite different 


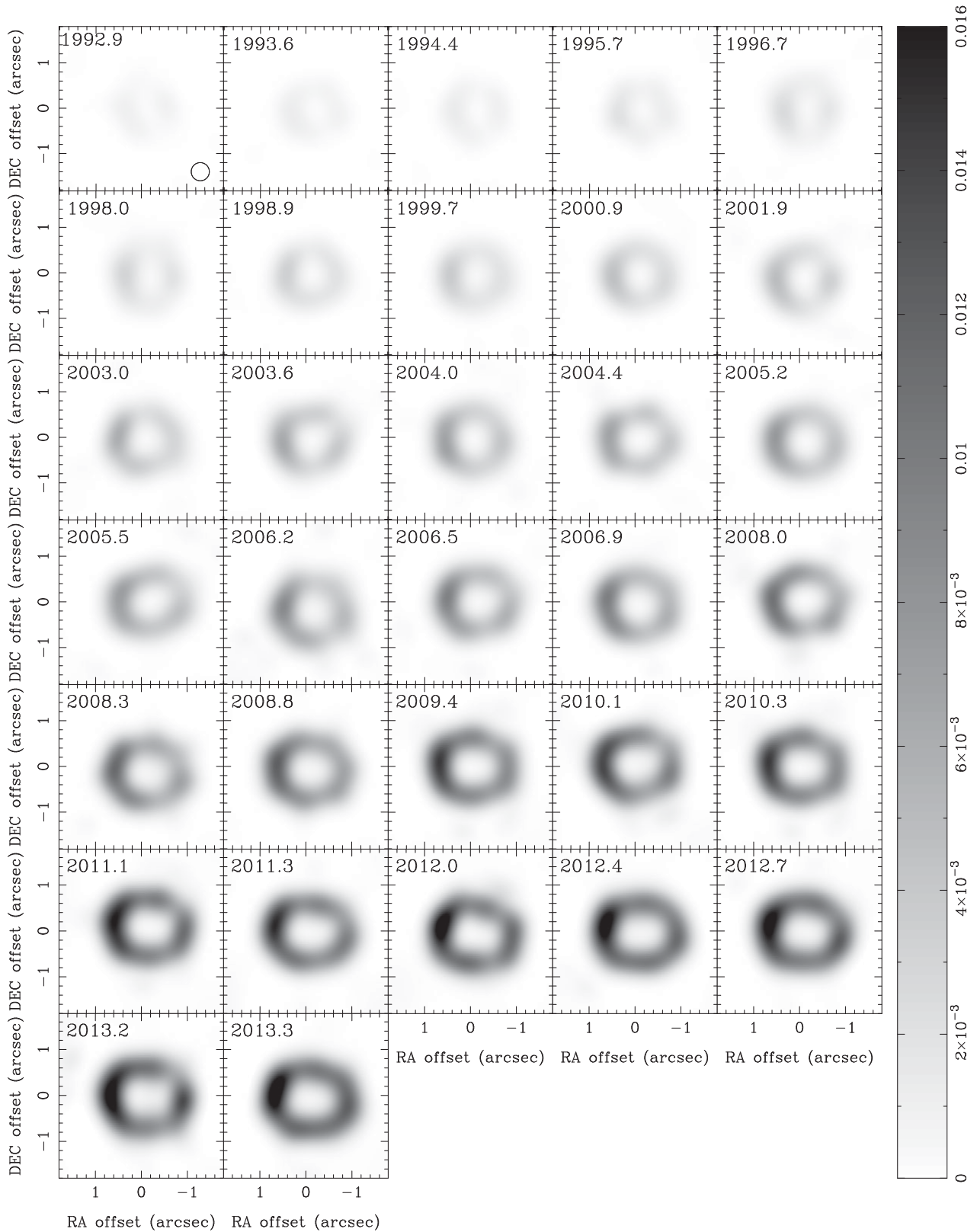

Figure 3. Radio observations at $9 \mathrm{GHz}$ have been conducted with ATCA at half-yearly or yearly intervals since the SN1987A remnant was first resolved in 1992. The above panels illustrate: (a) the strengthening radio emission; (b) the change from a circular (torus) to an elliptical (equatorial ring) morphology; and (c) the increasing diameter of the remnant. The year (CE) of the observation is listed in the top-left corner of each panel. Note the asymmetric nature of the remnant. Data from Ng et al. (2013, in preparation).

(Helder et al. 2013). This is now understood to be due to the different emission regions and the dominance of X-ray thermal radiation over synchrotron radiation dominant at radio wavelengths. 

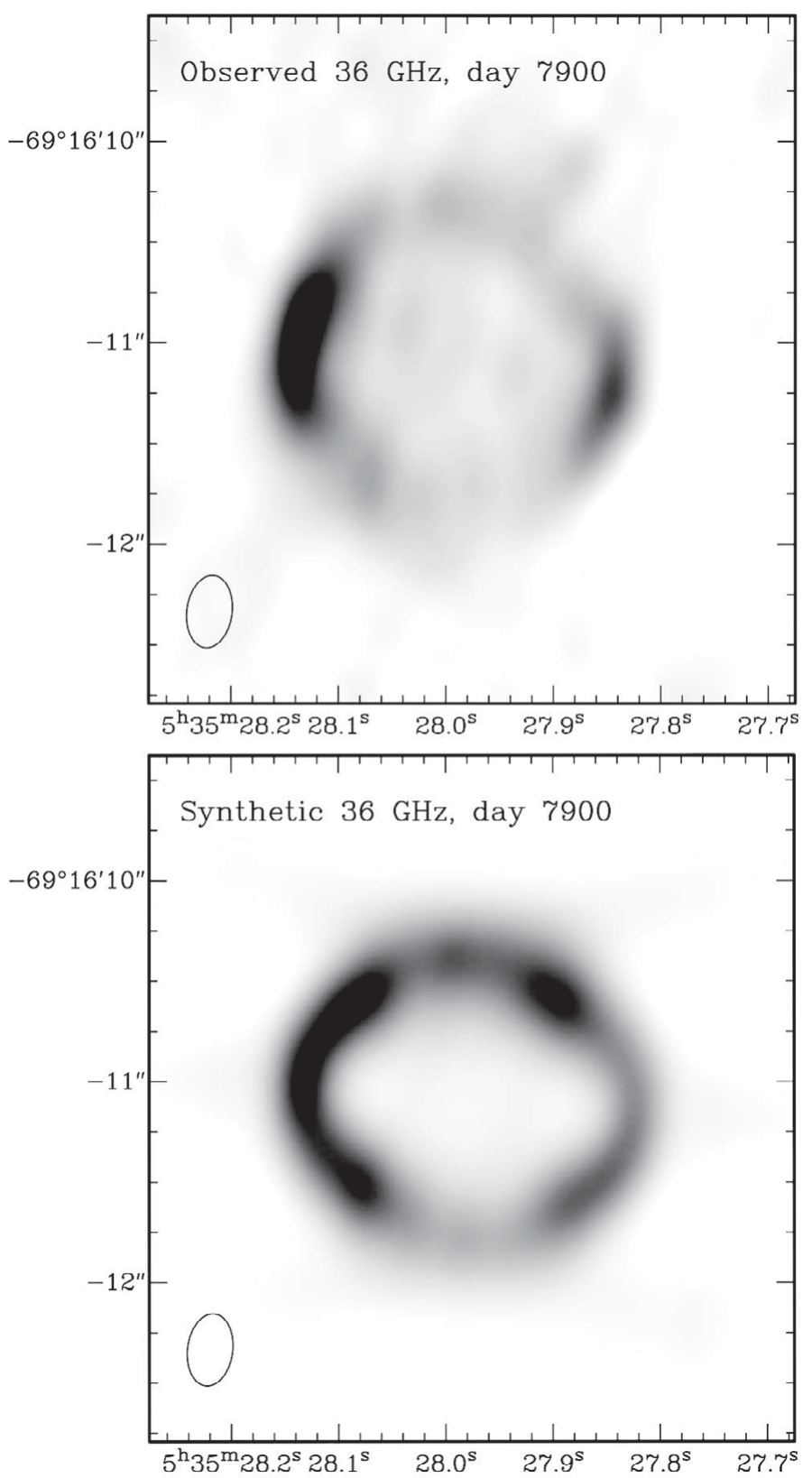

Figure 4. Top: A 36-GHz ATCA image of the radio remnant of SN1987A on day 7900 (Potter et al. 2009). Bottom: multi-dimensional hydrodynamic DSA model of the remnant at the same frequency and on the same day (Potter 2012).

Interpretation of the radio light curve has been assisted by high-resolution radio observations with the Australia Telescope Compact Array (ATCA), particularly when the remnant became strong enough to apply the technique of 'super-resolution'. This allows a resolution increase by a factor of 1.5 to 2 for a compact source like SN1987A with the available signal-to-noise ratio. A compilation of all $9 \mathrm{GHz}$ full uv-coverage ATCA images 
is shown in Fig. 3. Firstly, the rapid brightening of the whole remnant is apparent. This reflects the radio light curve shown in Fig. 2, although at $9 \mathrm{GHz}$ the increase is slightly steeper, reflecting the flattening of the radio spectrum over time. Secondly, on close inspection, the growth in the size of the remnant can be measured, reflecting the current expansion rate of $4000 \mathrm{~km} \mathrm{~s}^{-1}$ ( $\mathrm{Ng}$ et al. 2008). Notably, the expansion rate was much higher prior to switch-on of the radio emission at day 1200. This difference is explained as an inner cavity in models of the pre-supernova medium as shown in Fig. 1. Finally, it is apparent that the remnant is one-sided, with the eastern half being consistently $\sim 30 \%$ brighter until about 2007, after which the asymmetry decreases. The most likely explanation of this is explosion asymmetry.

\section{Models}

A number of hydrodynamic models have explored the propagation of the SN1987A shock front through the circumstellar medium. These have been used to predict dates for the collision of the shock front with the ring (Borkowski et al. 1997) and have also been used to explain the radio evolution of the remnant (Berezhko \& Ksenofontov 2006). However, most simulations have been one-dimensional and do not capture the inherent asymmetry of the pre-supernova environment. We have therefore attempted to model the hydrodynamical propagation of the shock front in three dimensions. We do this using an initial model which was motivated by observations and itself created from a plausible hydrodynamic evolution for a combined red/blue supergiant system. The initial model is that of Fig. 1. Further details (densities, temperatures, dimensions) are given in Potter (2012).

The radio emission is calculated from the hydrodynamic simulation using sub-grid physics, although it was not possible to include any back-reaction from the cosmic rays onto the shock front. Thermal electron are injected into the shock and assumed to be accelerated to high energies by the process of Diffusive Shock Acceleration (DSA). The electron energy spectrum and the magnetic field energy are used to calculate the synchrotron emissivity, which adiabatically decays after advection from the shock. As the simulations are in three dimensions, we can create synthetic radio images at any time and frequency. An example is the synthetic $36 \mathrm{GHz}$ image at day 7900, which is plotted alongside an actual ATCA image from Potter et al. (2009) at the same frequency in Fig.4. Parameters such as diameter, asymmetry and axis ratio can be calculated, and seem to accurately reproduce the past evolution of the remnant. Future predictions (Potter et al. in preparation) include a reversal of the asymmetry of the remnant. However, due to the highly non-linear nature of the DSA mechanism, it is more challenging to reproduce the exact shape of the radio light curve of Fig. 2. Nonetheless, our best fit suggests that the injection rate of those thermal electrons which are accelerated to cosmic ray energies must be around $4 \%$. This is remarkably efficient and gives an insight into the importance of SNRs as sources of highly energetic particles in galaxies.

\section{Future}

SNRs are believed to be important sources of cosmic rays in galaxies. These particles are important in facilitating the conversion of atomic gas into molecular form by providing charge to dust grains deeply embedded in these clouds. They also provide interstellar pressure that slows the accretion of cool gas onto galaxies. Cosmic ray acceleration processes can be further studied by tracking the evolution of the radio emission at the forward shock during the ongoing transition of SN1987A into an SNR. 
High energy particles are also created by magnetised spinning neutron stars. Although no pulsar has yet been detected, it is believed that a neutron star was created during the initial explosion. Evidence for this comes from the $\sim 21$ non-background neutrinos seen by the Kamiokande II, IMB and Baksan detectors about 2 hrs before the visible flash (Vissani et al. 2010). Zanardo et al. (2013) suggest that the conditions may now be favourable for detection of a pulsar. Indeed, tentative signs of a flat spectrum central component have been found in previous multi-frequency radio studies (Potter et al. 2009; Zanardo et al. 2013).

Finally, sub-mm observations indicate the presence of large amounts of cold dust in the ejecta of SN1987A (Matsuura et al. 2010). This is exciting as it implies that dust formation in supernovae is probably more important than previously believed, and perhaps resolves the puzzle of the rapid appearance of large quantities of dust at high redshift. Although not the subject of this paper, multi-frequency and spectral-line observations with ALMA will be crucial in the measurement of dust and gas mass and temperature, gas kinematics and in-situ dust and molecule formation.

\section{Acknowledgements}

Parts of this research were conducted by the Australian Research Council Centre of Excellence for All-sky Astrophysics (CAASTRO), through project number CE110001020.

\section{References}

Berezhko, E. G. \& Ksenofontov, L. T. 2006, ApJ, 650, 59

Borkowski, K. J., Blondin, J. M., \& McCray, R. 1997, ApJ, 476, 31

Chevalier, R. A. $1982, A p J, 259,302$

Chevalier, R. A. \& Dwarkadas, V. V. 1995, ApJ, 452, L45

Crotts, A. P. S., Kunkel, W. E., \& McCarthy, P. J. 1989, ApJ, 347, 61

Helder, E. A., Broos, P. S., Dewey, D., Dwek, E., McCray, R., Park, S., Racusin, J. L., Zhekov, S. A., \& Burrows, D. N. 2013, ApJ, 764, 11

Manchester, R. N., Gaensler, B. M., Wheaton, V. C., Staveley-Smith, L., Tzioumis, A. K., Bizunok, N. S., Kesteven, M. J., \& Reynolds, J. E. 2002, PASA, 19, 207

Matsuura, M. et al. 2010, Science, 333, 1258

Ng, C.-Y., Gaensler, B. M., Staveley-Smith, L., Manchester, R. N., Kesteven, M. J., Ball, L., \& Tzioumis, A. K. 2008, ApJ, 684, 481

Panagia, N., Gilmozzi, R., Macchetto, F., Adorf, H.-M., \& Kirshner, R. P. 1991, ApJ, 380, 23

Potter, T. M. 2012, Radio Observations and Multi-dimensional Simulations of the Expanding Remnant of SN1987A, Ph.D thesis, University of Western Australia

Potter, T. M., Staveley-Smith, L., Ng, C.-Y., Ball, L., Gaensler, B. M., Kesteven, M. J., Manchester, R. N., Tzioumis, A. K., \& Zanardo, G. 2009, ApJ, 705, 261

Staveley-Smith, L., Manchester, R. N., Kesteven, M. J., Reynolds, J. E., Tzioumis, A. K., Killeen, N. E. B., Jauncey, D. L., Campbell-Wilson, D., Crawford, D. F., \& Turtle, A. J. 1992, Nature, 355, 147

Storey, M. C. \& Manchester, R. N. 1987, Nature, 329, 421

Turtle, A. J., Campbell-Wilson, D., Bunton, J. D., Jauncey, D. L., Kesteven, M. J., Manchester, R. N., Norris, R. P., Storey, M. C., \& Reynolds, J. E. 1987, Nature, 327, 38

Vissani, F., Costantini, M. L., Fulgione, W., Ianni, A., \& Pagliaroli, G. 2010, in Frontier Objects in Astrophysics and Particle Physics, 2010 Vulcano workshop, arXiv:1008.4726

Wampler, E. J., Wang, L., Baade, D., Banse, K., D'Odorico, S., Gouiffes, C., \& Tarenghi, M. 1990, ApJ, 362, 13

Zanardo, G., Staveley-Smith, L., Ball, L., Gaensler, B. M., Kesteven, M. J., Manchester, R. N., Ng, C.-Y., Tzioumis, A. K., \& Potter, T. M. 2010, ApJ, 710, 1515 
Zanardo, G., Staveley-Smith, L., Ng, C.-Y., Gaensler, B. M., Potter, T. M., Manchester, R. N., \& Tzioumis, A. K. 2013, ApJ, 767, 98

\section{Discussion}

Chakraborti: Do you see the spectral index change with time?

StaVEley-Smith: The spectral index has flattened considerably from an initial value of -1 to the current value of $-0.7\left(S \propto \nu^{\alpha}\right)$ - see Zanardo et al. (2010).

BARTEL: What is your current limit on the spectral luminosity of any pulsar wind nebula that may exist in the center of SN1987A, e.g. in terms of the spectral luminosity of the Crab Nebula?

Staveley-Smith: The limit at frequencies of $8 \mathrm{GHz}$ and higher for a Central Nebula or pulsar is of the order of 1 mJy. This corresponds to a small fraction $(\sim 1 \%)$ of the spectral power of the Crab Nebula.

WANG: Do you have any handle on the total mass of the ring?

StaVELEY-Smith: Not really. The synchrotron component arises in the lower density components. There are some constraints on the ionized gas mass. 